\title{
Newly diagnosed glioblastoma in geriatric $(65+)$ patients: impact of patients frailty, comorbidity burden and obesity on overall survival
}

\author{
Matthias Schneider ${ }^{1}\left[\right.$ - Anna-Laura Potthoff ${ }^{1}$ - Elisa Scharnböck ${ }^{1} \cdot$ Muriel Heimann ${ }^{1}$ - Niklas Schäfer ${ }^{2}$. \\ Johannes Weller ${ }^{2}$. Christina Schaub ${ }^{2}$. Andreas H. Jacobs ${ }^{3}$. Erdem Güresir ${ }^{1}$. Ulrich Herrlinger ${ }^{2} \cdot$ Hartmut Vatter $^{1}$. \\ Patrick Schuss ${ }^{1}$
}

Received: 30 July 2020 / Accepted: 16 September 2020 / Published online: 29 September 2020

(c) The Author(s) 2020

\begin{abstract}
Object Increasing age is a known negative prognostic factor for glioblastoma. However, a multifactorial approach is necessary to achieve optimal neuro-oncological treatment. It remains unclear to what extent frailty, comorbidity burden, and obesity might exert influence on survival in geriatric glioblastoma patients. We have therefore reviewed our institutional database to assess the prognostic value of these factors in elderly glioblastoma patients.

Methods Between 2012 and 2018, patients aged $\geq 65$ years with newly diagnosed glioblastoma were included in this retrospective analysis. Patients frailty was analyzed using the modified frailty index (mFI), while patients comorbidity burden was assessed according to the Charlson comorbidity index (CCI). Body mass index (BMI) was used as categorized variable. Results A total of 110 geriatric patients with newly diagnosed glioblastoma were identified. Geriatric patients categorized as least-frail achieved a median overall survival (mOS) of 17 months, whereas most frail patients achieved a mOS of 8 months $(p=0.003)$. Patients with a CCI $>2$ had a lower mOS of 6 months compared to patients with a lower comorbidity burden (12 months; $p=0.03)$. Multivariate analysis identified "subtotal resection" $(p=0.02)$, "unmethylated MGMT promoter status" ( $\mathrm{p}=0.03)$, "BMI $<30$ " ( $\mathrm{p}=0.04)$, and "frail patient $(\mathrm{mFI} \geq 0.27)$ " $(\mathrm{p}=0.03)$ as significant and independent predictors of 1-year mortality in geriatric patients with surgical treatment of glioblastoma (Nagelkerke's $\mathrm{R}^{2} 0.31$ ).

Conclusions The present study concludes that both increased frailty and comorbidity burden are significantly associated with poor OS in geriatric patients with glioblastoma. Further, the present series suggests an obesity paradox in geriatric glioblastoma patients.
\end{abstract}

Keywords Geriatric glioblastoma patients $\cdot$ Frailty $\cdot$ Comorbidity $\cdot$ Survival

\section{Introduction}

Electronic supplementary material The online version of this article (https://doi.org/10.1007/s11060-020-03625-2) contains supplementary material, which is available to authorized users.

Matthias Schneider

matthias.schneider@ukbonn.de

1 Department of Neurosurgery, University Hospital Bonn, Venusberg-Campus 1, 53127 Bonn, Germany

2 Division of Clinical Neurooncology, Department of Neurology, University Hospital Bonn, Bonn, Germany

3 Department of Geriatric Medicine and Neurology, Johanniterkrankenhaus and CIO Bonn, Bonn, Germany
Although maximally safe surgery and adjuvant therapy have been the standard of care in glioblastoma treatment for many years, the management of elderly patients remains a challenge due to the increased incidence of treatment-related toxicities and slower recovery rates [1-5].

The challenge for optimal treatment of glioblastoma in geriatric patients resides in the balance between maximum radicality and reduction of intervention-related adverse events, which must be determined individually. Therefore, for elderly patients, specific aspects are important for a better assessment of treatment progression/success, such as a more detailed assessment of physical resources and function before each treatment. 
There is scarce data available on health status, physical resources, frailty and comorbidity burden as well as their implications for survival in older patients with glioblastoma.

Therefore, we have analyzed our institutional database with regard to a potential impact of the abovementioned pre-, peri- as well as immediately postoperatively collectable parameters on the success of treatment in geriatric patients with newly diagnosed glioblastoma.

\section{Materials and methods}

\section{Patients}

All patients with newly diagnosed glioblastoma who were operated on at the authors' facility between 2012 and 2018 were entered into a computerized database (SPSS, version 25 , IBM Corp., Armonk, NY). Only patients aged $\geq 65$ years who underwent surgical resection for newly diagnosed glioblastoma were included in the further analysis. Approval for this study was granted by the institutional ethics committee.

Information, including patient characteristics, radiological features, methylation status of the MGMT promoter, body mass index (BMI), functional neurological status at admission and during the course of treatment was recorded and further analyzed. The Karnofsky performance score (KPS) was used to evaluate geriatric patients according to their neurological functional status. In this context, KPS $\geq 70$ was defined as a favorable outcome during postoperative follow-up immediately after surgery and 3 and 12 months postoperatively. Patients frailty was analyzed using the modified frailty index (mFI), while patients comorbidity burden was assessed according to the Charlson comorbidity index (CCI).

Treatment decisions were made at the initial presentation of the patient and during follow-up by the institutional interdisciplinary tumor board meetings of the Center of Neurooncology, as described previously [6].

The extent of resection (EOR) was assessed in early $(<72 \mathrm{~h})$ postoperative magnetic resonance imaging (MRI, $3 \mathrm{~T}$ ). Gross-total resection (GTR) was determined as complete removal of the contrast-enhancing tissue (i.e. absence of residual enhancing tumor tissue).

Overall survival (OS) was measured from the day of glioblastoma surgery until death or last observation. All parameters were compared in terms of OS.

\section{Modified frailty index and CCI}

The Canadian Study on Health and Aging has developed a standardized frailty index (CSHA-FI) based on a cumulative deficit model. The CSHA-FI was linked to eleven variables from the database of the American Surgeons National Surgical Quality Improvement Program (NSQIP) to create a modified frailty index (mFI) [7]. The mFI thus contains the following 11 items: diabetes; functional status (not independent); chronic obstructive pulmonary disease or pneumonia; congestive heart failure; history of myocardial infarction; hypertension requiring medication; peripheral vascular disease or resting pain; impaired sensory function; transient ischaemic attack or cerebrovascular event in the medical history; previous cerebrovascular accident with neurological deficit; previous percutaneous coronary intervention, previous coronary surgery, or angina pectoris. For the calculation of the index, each item was allocated the same weight ( 1 point). The $\mathrm{mFI}$ was then computed for a given individual patient with total points as the sum of all items divided by 11 [8]. Although the $\mathrm{mFI}$ is not intended to be a dichotomized variable, patients were divided into three groups according to their $\mathrm{mFI}$ based on previous data: "least-frail" (mFI 0-0.08), "moderatelyfrail" ( $\mathrm{mFI} 0.09-0.26)$, and "frailest" $(\mathrm{mFI} \geq 0.27)$ [9].

Based on retrospective review of medical records, the preoperative comorbidity burden of included geriatric patients with glioblastoma was indexed by the CCI, as described elsewhere [10]. Data for the mFI and CCI was obtained from medical chart reviews as first listed diagnoses or derived from administrative systems using the 10th revision of the International Statistical Classification of Diseases and Related Health Problems (ICD-10) codes [11]. These codes were manually examined by two coding experts and a general physician for face validity. According to the current literature and for better clinical applicability, the analyzed geriatric patients with glioblastoma were separated into two groups according to their calculated CCI: CCI 0-2 and CCI > $2[12,13]$.

\section{Statistics}

Data analysis was performed using the computer software package SPSS (version 25, IBM Corp., Armonk, NY). Unpaired categorical and binary variables were analyzed in contingency tables using the Fisher's exact test. The Mann-Whitney U-test was chosen to compare continuous variables as the data were mostly not normally distributed. OS was analyzed by the Kaplan-Meier method using Gehan-Breslow-Wilcoxon test. Results with $\mathrm{p}<0.05$ were considered statistically significant.

Furthermore, a backward stepwise method was used to construct a multivariate logistic regression model in order to find independent predictors of 1-year mortality in elderly patients with glioblastoma who underwent surgical resection. 


\section{Results}

\section{Patient characteristics}

Between 2012 and 2018, a total of 110 geriatric patients underwent surgery for newly diagnosed glioblastoma at the Department of Neurosurgery, University Hospital Bonn. The

Table 1 Patient and tumor characteristics

\begin{tabular}{ll}
\hline & $\mathrm{n}=110$ \\
\hline Median age at operation $(95 \% \mathrm{CI})$ & $72(65-86)$ \\
Female sex & $46(42 \%)$ \\
Median preoperative KPS (95\% CI) & $80(50-100)$ \\
Median BMI (95\% CI) & $26.1(19.6-44.1)$ \\
CCI 0-2 & $92(84 \%)$ \\
CCI $>2$ & $18(16 \%)$ \\
Median mFI & 0.18 \\
Preoperative anticoagulation/antiplatelet & $51(46 \%)$ \\
medication & \\
Tumor-related epilepsy & $22(20 \%)$ \\
MGMT methylated & $48(44 \%)$ \\
MGMT unmethylated & $57(52 \%)$ \\
MGMT not available & $5(5 \%)$ \\
IDH wild type & $97(88 \%)$ \\
IDH mutant & $7(6 \%)$ \\
IDH not availbale & $6(6 \%)$ \\
Adjuvant chemotherapy & $83(75 \%)$ \\
Adjuvant radiotherapy & $94(85 \%)$ \\
mOS (months) & $11(95 \%$ CI 9.4-12.6) \\
\hline
\end{tabular}

$B M I$ body mass index, $C C I$ Charlson comorbidity index, $C I$ confidence interval, $I D H$ isocitrate dehydrogenase, KPS Karnofsky performance score, $m F I$ modified frailty index, $M G M T$ O-6-methylguanineDNA methyltransferase, $m O S$ median overall survival, $S D$ standard deviation median age was 72 years (range $65-86$ years). Geriatric glioblastoma patients exhibited a median preoperative KPS of 80 (range 50-100). Tumors most frequently involved the temporal lobe $(36 \%)$, followed by the frontal $(28 \%)$, parietal (23\%), and occipital (11\%) regions. Multifocal findings were present in 6 patients (6\%). GTR was performed in 66 patients $(60 \%)$, STR in 44 patients $(40 \%)$. Median OS for geriatric patients with glioblastoma was 11 months $(95 \% \mathrm{CI}$ 9.4-12.6). Further details are given in Table 1.

\section{Influence of body mass index on overall survival}

Geriatric glioblastoma patients presented with a median BMI of 26.1 (range 19.6-44.1). 39 patients (36\%) met the classification of a normal weight $(\mathrm{BMI}<25), 43$ patients (39\%) were overweight (BMI 25-29.9), 28 patients (25\%) appeared to be obese $(\mathrm{BMI} \geq 30)$. Patients classified as normal weighted had a mOS of 10 months (95\% CI 7.4-12.6), overweight patients had an mOS of 9 months $(95 \%$ CI 6.4-11.7) and obese patients had a mOS of 15 months (95\% CI 12.9-17.1; Fig. 1). Patients with a BMI $<30$ had a mPFS of 7 months (95\% CI 6-9), patients with a BMI $\geq 30$ had a mPFS of 12 (95\% CI 7-25; $p=0.036$, Supplementary Figure S1a).

\section{Influence of comorbidity burden on overall survival}

Geriatric glioblastoma patients exhibited a median preoperative CCI of 0.5. Detailed frequencies of conditions included in the CCI are given in Table 2. Patients with a CCI $\geq 2$ achieved a mOS of 5 months (95\% CI 0-10.5), while patients with a lower comorbidity index achieved a mOS of 12 months (95\% CI 10.5-13.5; $\mathrm{p}=0.012$, Fig. 2). Patients with a CCI $>2$ achieved a mPFS of 6 months (95\% CI 2-12), while patients with a lower comorbidity index achieved a
Fig. 1 Obesity correlates to prolonged overall survival rates. a Kaplan-Meier curves for OS of geriatric glioblastoma patients stratified according to normal weight (BMI 18-24.9 kg/m²), overweight (BMI 25-29.9 kg/ $\left.\mathrm{m}^{2}\right)$ and obese $(\mathrm{BMI} \geq 30 \mathrm{~kg} /$ $\mathrm{m}^{2}$ ). b Scatter plots depict median and distribution of OS dependent on the BMI-levels indicated $B M I$ body mass index, $O S$ overall survival
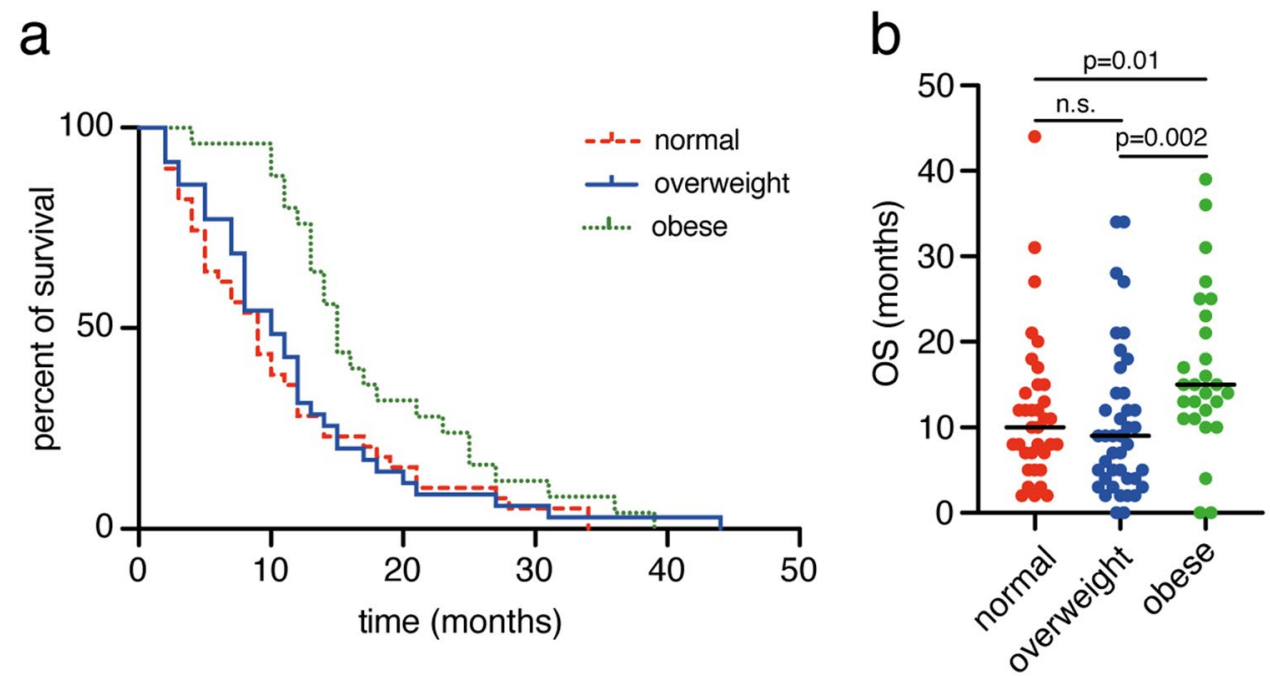
Table 2 Frequency of Charlson comorbidity index conditions $(\mathrm{n}=110)^{\mathrm{a}}$

\begin{tabular}{llc}
\hline Index weight & Condition & Frequency $\%(\mathrm{n})$ \\
\hline 1 & Coronary artery disease & $6.4(7)$ \\
1 & Congestive heart failure & $3.6(4)$ \\
1 & Peripheral vascular disease & $0.9(1)$ \\
1 & Cerebrovascular disease & $7.3(8)$ \\
1 & Dementia & $5.5(6)$ \\
1 & Chronic pulmonary disease & $5.5(6)$ \\
1 & Connective tissue disease & $0(0)$ \\
1 & Ulcer disease & $2.7(3)$ \\
1 & Mild liver disease & $1.8(2)$ \\
1 & Diabetes & $13.6(15)$ \\
2 & Hemiplegia & $7.3(8)$ \\
2 & Moderate/severe renal disease & $4.5(5)$ \\
2 & Diabetes with end-organ damage & $0(0)$ \\
2 & Any tumor & $16.4(18)$ \\
2 & Leukemia & $0.9(1)$ \\
2 & Lymphoma & $0(0)$ \\
3 & Moderate/severe liver disease & $0.9(1)$ \\
6 & Metastatic solid tumor & $0.9(1)$ \\
6 & AIDS & $0(0)$ \\
\hline
\end{tabular}

${ }^{a}$ Values represent number of patients unless otherwise indicated (\%)

mPFS of 8 months (95\% CI 7-12; $p=0.014$, Suplementary Figure S1b).

\section{Influence of patients frailty on overall survival}

At preoperative admission status, geriatric glioblastoma patients presented with a median $\mathrm{mFI}$ of 0.18 . Detailed frequencies of conditions included in the $\mathrm{mFI}$ are given in Table 3. Analysis of mortality-rates revealed a considerable higher probability of death with increasing index (Fig. 3). After categorization, geriatric patients defined as least-frail and moderately-frail achieved a mOS of 13 months, whereas the mot frail patients achieved a mOS of 7 months ( $<<0.0001$, Fig. 3a,b). For the group of patients aged $65-75$ years, patients with $\mathrm{mFI}<0.27$ exhibited a mOS of 16 months, compared to 11 months for patients with $\mathrm{mFI} \geq 0.27(\mathrm{p}=0.007)$. For the group of patients aged $76-85$ years, respective values for $\mathrm{mOS}$ were 11 months vs 5.5 months $(\mathrm{p}=0.03)$ (Fig. 3c).

Patients with a $\mathrm{mFI}<0.27$ exhibited a mPFS of 8 months (95\% CI 7-12), patients with a $\mathrm{mFI} \geq 0.27$ had a mPFS of 4 months (95\% CI 2-7; $<<0.0004$, Supplementary Figure S1c).

\section{Multivariate analysis}

We conducted a multivariate logistic regression analysis to identify independent predictors of 1-year mortality in geriatric patients with glioblastoma. The multivariate analysis identified "subtotal resection" $(\mathrm{p}=0.02$, OR $3.1,95 \% \mathrm{CI}$ 1.2-7.7), "unmethylated MGMT promoter methylation status" ( $\mathrm{p}=0.03$, OR $2.7,95 \%$ CI $1.1-6.5)$, "BMI $<30$ " $(\mathrm{p}=0.04$, OR 3.1, 95\% CI 1.1-9.2), and "frail patient classification $(\mathrm{mFI} \geq 0.27) "(\mathrm{p}=0.03, \mathrm{OR} 3.2,95 \%$ CI $1.1-8.8)$ as significant and independent predictors of 1-year mortality (Nagelkerke's $\mathrm{R}^{2}$ 0.31).

\section{Discussion}

As the incidence of glioblastoma in elderly patients increases as this population grows, it has become increasingly important to identify effective treatment regimens that might extend survival in this vulnerable patient population [14]. Older patients with glioblastoma are often treated more conservatively than younger ones [15]. Several studies have noted that older patients with glioblastoma often only receive biopsy or scaled down adjuvant therapy $[15$,
Fig. 2 Comorbidity burden correlates to limited overall survival rates. a Kaplan-Meier curves for OS stratified into geriatric glioblastoma patients with $\mathrm{CCI}<2$ and $\leq 2$. b Scatter plots depict median and distribution of OS dependent on the CCI-levels indicated $C C I$ Charlson comorbidity index, $O S$ overall survival a

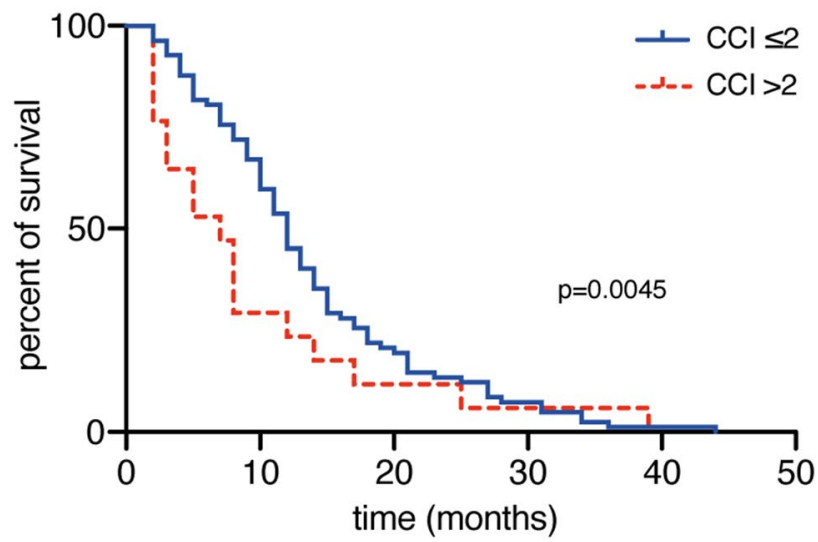

b

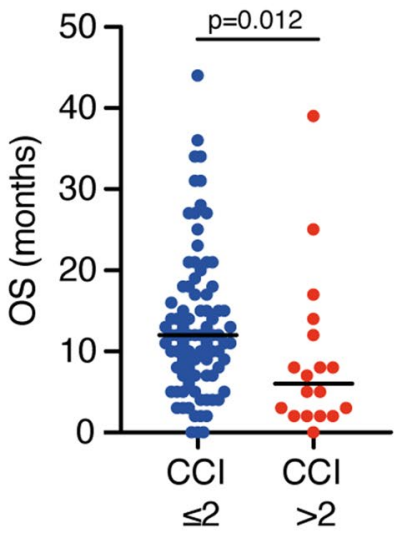


Table 3 Frequency of patient frailty according to the modified frailty index $(\mathrm{n}=110)$

\begin{tabular}{llc}
\hline Index weight & Description & Frequency \% (n) \\
\hline 1 & Functional health status prior surgery (only dependent) & $36.4(40)$ \\
1 & History of diabetes mellitus & $13.6(15)$ \\
1 & History of severe COPD/current pneumonia & $8.2(9)$ \\
1 & Congestive heart failure & $3.6(4)$ \\
1 & History of myocardial infarction & $6.4(7)$ \\
1 & Previous percutaneous coronary intervention; previous cardiac & $34.5(38)$ \\
& surgery; history of angina & \\
1 & Hypertension requiring medication & $67.3(74)$ \\
1 & Impaired sensorium & $5.5(6)$ \\
1 & History of transient ischemic attack & $4.5(5)$ \\
1 & Cerebrovascular accident/stroke with neurologic deficit & $2.7(3)$ \\
\hline
\end{tabular}

b

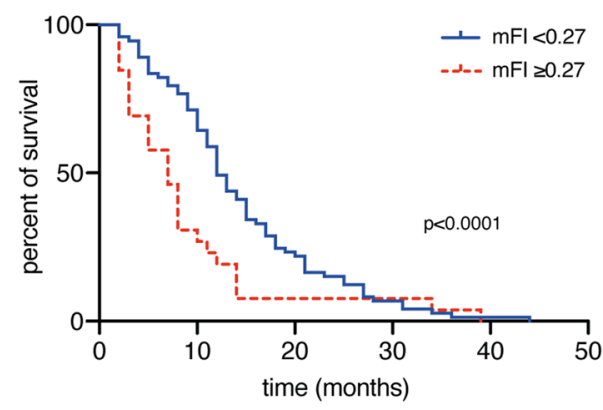

C

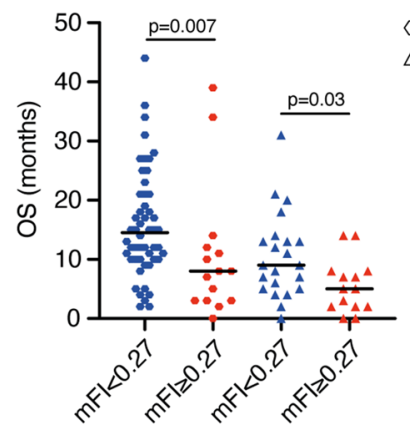

65-75 years

$\triangle 76-85$ years plots depict median and distribution of OS dependent on the mFI-levels for further stratification by age as indicated. $m F I$ modified frailty index, $O S$ overall survival

In Contrary to a reported positive influence of elevated BMI levels on survival in patients with glioblastoma averaged over all age groups, various studies provide partially contradictory data [22-24]. This underlines the divergent character of BMI as a measure of obesity in patients with underlying solid malignancies. On the one hand, obesity is associated with a poor prognosis in certain oncological scenarios due to increased circulating concentrations of metabolic and pro-inflammatory hormones that might stimulate tumor growth and metastatic spread [23]. Further hypotheses for the association between obesity and poor outcome include uncontrolled hyperglycemia in obese patients, which may promote tumor growth, and/or suboptimal chemotherapy due to dosage thresholds [22]. On the other hand, it has been suggested that an increased BMI is also associated with a larger muscle mass, which in patients with advanced malignant disease serves as a potent energy source and thus results in a better prognosis [25]. 


\section{Influence of patient frailty on survival}

In geriatric research, assessing the frailty of elderly patients is an important instrument for predicting morbidity and mortality [26]. In a systematic review of the literature, Pazniokas et al. were able to illustrate that frailty is associated with poor outcomes in a variety of neurosurgical disorders [20]. At the same time, the authors note that the neurosurgical literature is extremely heterogeneous in its methodological assessment of frailty. Following the recommendation of Pazniokas and co-workers, the assessment of frailty in the present study is based on the application of the modified frailty index [7]. Increased frailty of an elderly glioblastoma patient results in a significantly higher probability of poorer survival. We were able to determine the influence of frailty by using the Kaplan-Meier method in terms of overall survival $(\mathrm{p}=0.005)$ as well as applying a multivariate analysis of 1 -year mortality $(p=0.03)$ in our patient cohort.

The relevance of these results, especially with regard to the influence of the frailty of elderly patients with glioblastoma on outcome, should not be underestimated. According to a recent study of Rahmani et al. surgery for glioblastoma in the eldely consitutes a safe treatment modality resulting in low death and life-threatening morbidity [27]. However, the authors were able to show that about $35 \%$ of patients experienced a change in living disposition postoperatively. Thus, the authors conclude that understanding the rates and risk factors for adverse events following glioblastoma resection should guide neurosurgeons in treatment decision in this selected patient cohort [27]. Here, we propose the $\mathrm{mFI}$ is quite an easy to use screening method that not only enables to preoperatively select for high-risk patients that might require special attention in surgical management and after care, but also might become crucial for optimizing individually tailored treatment strategies as well as counselling of patients and family members. Further multicenter-based studies are needed in order to become able to sufficiently cope with the challenges in the course of interdisciplinary modern treatment and aftercare in the geriatric glioblastoma patient cohort.

\section{Limitations}

The present study has multiple limitations. The data collection was conducted retrospectively. The patients were not randomized, but treated according to the preference of the treating physicians. Furthermore, the study utilizes the $\mathrm{mFI}$ as an assessment tool for frailty, which does not query all established factors of frailty. Nevertheless, the strength of the present work is found in the detailed, standardized collection of these data, the results of which should be supported by the initiation of multicenter prospective studies.

\section{Conclusions}

The present study is the first to demonstrate a distinct association between preoperatively increased frailty and decreased survival following surgical treatment of geriatric glioblastoma patients. The results of this study should contribute to a better assessment of the risk/benefit ratio for the treating physician, so that a different kind of focused attention during treatment course might arise and both patients and relatives might be better informed prior surgery. Thus, the authors emphasize that comprehensive assessment of geriatric patients with glioblastoma, including comorbidity burden, frailty and nutritional status, may help clinicians to develop suitable, appropriate and precise treatment strategies for these vulnerable patients.

Funding Open Access funding enabled and organized by Projekt DEAL.

\section{Compliance with ethical standards}

Conflict of interest The authors declare that the article content was composed in the absence of any commercial or financial relationship that could be construed as a potential conflict of interest.

Open Access This article is licensed under a Creative Commons Attribution 4.0 International License, which permits use, sharing, adaptation, distribution and reproduction in any medium or format, as long as you give appropriate credit to the original author(s) and the source, provide a link to the Creative Commons licence, and indicate if changes were made. The images or other third party material in this article are included in the article's Creative Commons licence, unless indicated otherwise in a credit line to the material. If material is not included in the article's Creative Commons licence and your intended use is not permitted by statutory regulation or exceeds the permitted use, you will need to obtain permission directly from the copyright holder. To view a copy of this licence, visit http://creativecommons.org/licenses/by/4.0/.

\section{References}

1. Wick A, Kessler T, Elia AEH, Winkler F, Batchelor TT, Platten M, Wick W (2018) Glioblastoma in elderly patients: solid conclusions built on shifting sand? Neuro Oncol 20:174-183. https://doi. org/10.1093/neuonc/nox 133

2. Keime-Guibert F, Chinot O, Taillandier L, Cartalat-Carel S, Frenay M, Kantor G, Guillamo JS, Jadaud E, Colin P, Bondiau PY, Menei P, Loiseau H, Bernier V, Honnorat J, Barrie M, Mokhtari K, Mazeron JJ, Bissery A, Delattre JY, Association of FrenchSpeaking N-O (2007) Radiotherapy for glioblastoma in the elderly. N Engl J Med 356:1527-1535. https://doi.org/10.1056/ NEJMoa065901

3. Perry JR, Laperriere N, O'Callaghan CJ, Brandes AA, Menten J, Phillips C, Fay M, Nishikawa R, Cairncross JG, Roa W, Osoba D, Rossiter JP, Sahgal A, Hirte H, Laigle-Donadey F, Franceschi E, Chinot O, Golfinopoulos V, Fariselli L, Wick A, Feuvret L, Back M, Tills M, Winch C, Baumert BG, Wick W, Ding K, Mason WP, 
Trial I (2017) Short-course radiation plus temozolomide in elderly patients with glioblastoma. N Engl J Med 376:1027-1037. https ://doi.org/10.1056/NEJMoa1611977

4. Malmstrom A, Gronberg BH, Marosi C, Stupp R, Frappaz D, Schultz H, Abacioglu U, Tavelin B, Lhermitte B, Hegi ME, Rosell J, Henriksson R, Nordic Clinical Brain Tumour Study Group (2012) Temozolomide versus standard 6-week radiotherapy versus hypofractionated radiotherapy in patients older than 60 years with glioblastoma: the Nordic randomised, phase 3 trial. Lancet Oncol 13:916-926. https://doi.org/10.1016/S1470-2045(12)70265-6

5. Wick W, Platten M, Meisner C, Felsberg J, Tabatabai G, Simon M, Nikkhah G, Papsdorf K, Steinbach JP, Sabel M, Combs SE, Vesper J, Braun C, Meixensberger J, Ketter R, Mayer-Steinacker R, Reifenberger G, Weller M, Society NOASGoN-oWGoGC (2012) Temozolomide chemotherapy alone versus radiotherapy alone for malignant astrocytoma in the elderly: the NOA-08 randomised, phase 3 trial. Lancet Oncol 13:707-715. https://doi.org/10.1016/ S1470-2045(12)70164-X

6. Schneider M, Potthoff AL, Keil VC, Güresir Á, Weller J, Borger V, Hamed M, Waha A, Vatter H, Güresir E, Herrlinger U, Schuss P (2019) Surgery for temporal glioblastoma: lobectomy outranks oncosurgical-based gross-total resection. J Neurooncol 145:143150. https://doi.org/10.1007/s11060-019-03281-1

7. Velanovich V, Antoine H, Swartz A, Peters D, Rubinfeld I (2013) Accumulating deficits model of frailty and postoperative mortality and morbidity: its application to a national database. J Surg Res 183:104-110. https://doi.org/10.1016/j.jss.2013.01.021

8. Tsiouris A, Hammoud ZT, Velanovich V, Hodari A, Borgi J, Rubinfeld I (2013) A modified frailty index to assess morbidity and mortality after lobectomy. J Surg Res 183:40-46. https://doi. org/10.1016/j.jss.2012.11.059

9. Cloney M, D'Amico R, Lebovic J, Nazarian M, Zacharia BE, Sisti MB, Bruce JN, McKhann GM, Iwamoto FM, Sonabend AM (2016) Frailty in geriatric glioblastoma patients: a predictor of operative morbidity and outcome. World Neurosurg 89:362-367. https://doi.org/10.1016/j.wneu.2015.12.096

10. Charlson ME, Pompei P, Ales KL, MacKenzie CR (1987) A new method of classifying prognostic comorbidity in longitudinal studies: development and validation. J Chronic Dis 40:373-383. https ://doi.org/10.1016/0021-9681(87)90171-8

11. Glasheen WP, Cordier T, Gumpina R, Haugh G, Davis J, Renda A (2019) Charlson comorbidity index: ICD-9 update and ICD-10 translation. Am Health Drug Benefits 12:188-197

12. Ening G, Osterheld F, Capper D, Schmieder K, Brenke C (2015) Charlson comorbidity index: an additional prognostic parameter for preoperative glioblastoma patient stratification. J Cancer Res Clin Oncol 141:1131-1137. https://doi.org/10.1007/s0043 2-014-1907-9

13. Giaccherini L, Galaverni M, Renna I, Timon G, Galeandro M, Pisanello A, Russo M, Botti A, Iotti C, Ciammella P (2019) Role of multidimensional assessment of frailty in predicting outcomes in older patients with glioblastoma treated with adjuvant concurrent chemo-radiation. J Geriatr Oncol 10:770-778. https://doi. org/10.1016/j.jgo.2019.03.009

14. Lorimer CF, Saran F, Chalmers AJ, Brock J (2016) Glioblastoma in the elderly-how do we choose who to treat? J Geriatr Oncol 7:453-456. https://doi.org/10.1016/j.jgo.2016.07.005

15. Babu R, Komisarow JM, Agarwal VJ, Rahimpour S, Iyer A, Britt D, Karikari IO, Grossi PM, Thomas S, Friedman AH, Adamson $\mathrm{C}$ (2016) Glioblastoma in the elderly: the effect of aggressive and modern therapies on survival. J Neurosurg 124:998-1007. https ://doi.org/10.3171/2015.4.JNS142200

16. Iwamoto FM, Reiner AS, Panageas KS, Elkin EB, Abrey LE (2008) Patterns of care in elderly glioblastoma patients. Ann Neurol 64:628-634. https://doi.org/10.1002/ana.21521

17. Rebagliati GA, Sciume L, Iannello P, Mottini A, Antonietti A, Caserta VA, Gattoronchieri V, Panella L, Callegari C (2016) Frailty and resilience in an older population. The role of resilience during rehabilitation after orthopedic surgery in geriatric patients with multiple comorbidities. Funct Neurol 31:171-177. https:// doi.org/10.11138/fneur/2016.31.3.171

18. Gijzel SMW, Whitson HE, van de Leemput IA, Scheffer M, van Asselt D, Rector JL, Olde Rikkert MGM, Melis RJF (2019) Resilience in clinical care: getting a grip on the recovery potential of older adults. J Am Geriatr Soc 67:2650-2657. https://doi. org/10.1111/jgs.16149

19. Ritt M, Ritt JI, Sieber CC, Gassmann KG (2017) Comparing the predictive accuracy of frailty, comorbidity, and disability for mortality: a 1-year follow-up in patients hospitalized in geriatric wards. Clin Interv Aging 12:293-304. https://doi.org/10.2147/ CIA.S 124342

20. Pazniokas J, Gandhi C, Theriault B, Schmidt M, Cole C, Al-Mufti F, Santarelli J, Bowers CA (2020) The immense heterogeneity of frailty in neurosurgery: a systematic literature review. Neurosurg Rev. https://doi.org/10.1007/s10143-020-01241-2

21. Haslam DW, James WP (2005) Obesity. Lancet 366:1197-1209. https://doi.org/10.1016/S0140-6736(05)67483-1

22. Siegel EM, Nabors LB, Thompson RC, Olson JJ, Browning JE, Madden MH, Han G, Egan KM (2013) Prediagnostic body weight and survival in high grade glioma. J Neurooncol 114:79-84. https ://doi.org/10.1007/s11060-013-1150-2

23. Jones LW, Ali-Osman F, Lipp E, Marcello JE, McCarthy B, McCoy L, Rice T, Wrensch M, Il'yasova D (2010) Association between body mass index and mortality in patients with glioblastoma mutliforme. Cancer Causes Control 21:2195-2201. https:// doi.org/10.1007/s10552-010-9639-x

24. Schneider M, Borger V, Grigutsch D, Guresir A, Potthoff AL, Velten M, Vatter H, Guresir E, Schuss P (2020) Elevated body mass index facilitates early postoperative complications after surgery for intracranial meningioma. Neurosurg Rev. https://doi. org/10.1007/s10143-020-01281-8

25. Caan BJ, Cespedes Feliciano EM, Kroenke CH (2018) The importance of body composition in explaining the overweight paradox in cancer-counterpoint. Cancer Res 78:1906-1912. https://doi. org/10.1158/0008-5472.CAN-17-3287

26. Hall DE, Arya S, Schmid KK, Carlson MA, Lavedan P, Bailey TL, Purviance G, Bockman T, Lynch TG, Johanning JM (2017) Association of a frailty screening initiative with postoperative survival at 30, 180, and 365 days. Jama Surg 152:233-240. https ://doi.org/10.1001/jamasurg.2016.4219

27. Rahmani R, Tomlinson SB, Santangelo G, Warren KT, Schmidt T, Walter KA, Vates GE (2020) Risk factors associated with early adverse outcomes following craniotomy for malignant glioma in older adults. J Geriatr Oncol 11:694-700. https://doi. org/10.1016/j.jgo.2019.10.019

Publisher's Note Springer Nature remains neutral with regard to jurisdictional claims in published maps and institutional affiliations. 